\title{
Impact De L'exploitation Artisanale De La Sabliere De Toutsang Sur La Structure Des Communautes De Macroinvertebres Du Cours D'eau Doulahang A Dschang (Ouest Cameroun)
}

\author{
Ngameni Tchamadeu Norbert \\ Asanga Patricia Fai \\ Mpoame Mbida
}

Laboratoire de Biologie et d'Ecologie Appliquées, Département de Biologie Animale, Faculté des Sciences, Université de Dschang, Cameroun

\section{Kenko NKontcheu Daniel Brice}

Zoology Laboratory, Department of Zoology and Animal Physiology, Faculty of Science, University of Buea, Cameroon

doi: 10.19044/esj.2017.v13n23p254 URL:http://dx.doi.org/10.19044/esj.2017.v13n23p254

\begin{abstract}
The impact of wastewaters from the Toutsang sandpit (Dschang), both on water parameters and on benthic macro-invertebrate community structure of the Doulahang stream was studied from March 2015 to February 2016 on a monthly basis in three sampling stations named TS1, TS2 and TS3. The physico-chemical analysis were carried out according to standard protocols while the benthic macro-fauna was harvested using a haze net of $30 \mathrm{~cm}$ side and $400 \mu \mathrm{m}$ mesh size. The analysis of physico-chemical parameters revealed that the introduction of wastewater from the sandpit at the TS2 station led to an increase of the temperature, conductivity, total dissolved solids, salinity, suspended matter, turbidity and colour. The wildlife inventory identified 63 taxa divided into 4 phyla, 6 classes, 13 orders and 31 families. The lowest values of the taxonomic richness (18 taxa, against 46 taxa and 47 taxa respectively to stations TS1 and TS3), the Shannon diversity index (2.48 bits / ind.) and the Pielou's evenness (0.62) were observed at station TS2 which received wastewaters from the sandpit. Similarly, the low values of the richness and abundance of the pollutionsensitive groups (Ephemeroptera-Trichoptera-Odonata) were recorded in this station. Significant negative correlations were found between some metrics (total taxonomic richness, taxonomic richness of the Diptera, Richness of Ephemeroptera-Trichoptera-Odonata, Taxonomic richness of insects, Relative abundance of Ephemeroptera-Trichoptera-Odonata, Shannon-
\end{abstract}


Weaver diversity index, Pielou's evenness) descriptors of the structure of benthic macro-fauna communities and physico-chemical parameters indicative of physical pollution such as turbidity, suspended matter and colour.

Keywords: Toutsang sand pit, Dcshang, water quality, physical pollution, benthic macroinvertebrates

\section{Résumé}

L'influence des eaux usées en provenance de la sablière de Toutsang à Dschang sur la qualité des eaux, la structure et la composition des assemblages des macroinvertébrés benthiques du cours d'eau Doulahang a été étudiée de mars 2015 à février 2016 selon un rythme mensuel dans trois stations d'échantillonnage dénommées TS1, TS2 et TS3. Les analyses physicochimiques ont été effectuées suivant les méthodes standards, tandis que la macrofaune benthique a été récoltéé à l'aide d'un filet troubleau de 30 $\mathrm{cm}$ de côté et $400 \mu \mathrm{m}$ d'ouverture de maille. L'analyse des paramètres physico-chimiques a révélé que l'introduction des eaux usées de la sablière au niveau de la station TS2 entraine une augmentation des valeurs de la température, de la conductivité, du taux de solides dissous, de la salinité, des matières en suspension, de la turbidité et de la couleur. L'inventaire faunique a permis d'identifier 63 taxons repartis entre 4 embranchements, 6 classes, 13 ordres et 31 familles. Les plus faibles valeurs de la richesse taxonomique (18 taxons, contre 46 et 47 taxons respectivement aux stations TS1 et TS3), de l'indice de diversité de Shannon (2,48 bits/ind.) et de l'équitabilité de Pielou $(0,62)$ ont été observées au niveau de la station TS2 qui reçoit les eaux usées de la sablière. De même, les faibles valeurs de la richesse et de l'abondance des groupes polluo-sensibles (Ephéméroptères-TrichoptèresOdonates) ont été enregistrées dans cette station. . Des corrélations négatives et significatives ont été notées entre certaines métriques (Richesse taxonomique totale, Richesse taxonomique des Diptères, Richesse des Ephéméroptère-Trichoptère-Odonate, Richesse taxonomique des Insectes, Abondance relative des Ephéméroptères-Trichoptère-Odonate, Diversité de Shannon et Weaver, Equitabilité de Pielou) descripteurs de la structure des communautés de la macrofaune benthique et les paramètres physicochimiques indicateurs de la pollution physique tels que la turbidité, les matières en suspension et la couleur.

Mots-clés: Sablière artisanale, Dschang, qualité des eaux, pollution physique, macroinvertébrés benthiques 


\section{Introduction}

A Dschang, le besoin accru de sable pour approvisionner le secteur du bâtiment et le coût élevé du sable alluvionnaire de Santchou ont poussés les populations à se lancer dans l'exploitation affenée des sablières. La sablière de Toutsang et l'une des plus grandes sablières de la ville de Dschang. Cette activité leur génère des devises, réduit le taux de chômage, contribue au développement de la ville de Dschang du fait de fort taux d'utilisation du sable produit dans la localité de Dschang et de ses environs. Cependant ces nombreux atouts ne vont pas sans impacts considérables sur l'environnement. L'activité de la sablière entraine une augmentation de la turbidité et des matières en suspension (Kikouagni, 2015). Elle augmente également le transfert de sédiments fins (sable, argile, limons) vers les cours d'eau (Gayraud et al., 2002). Ces sédiments fins, principalement transportés en suspension, ont de nombreuses conséquences sur les cours d'eau. Ils peuvent entrainer un colmatage du lit des cours d'eau et, par voie de conséquences, des effets dommageables sur la biocénose aquatique en colmatant les habitats et les frayères de la faune benthique (Greig et al., 2005 ; Rabeni et al., 2005 ; Bo et al., 2007 ; Bilotta et Brazier, 2008 ; Kemp et al., 2011 ;). Les principaux effets du colmatage sont donc à la fois physique (modification et uniformisation des faciès d'écoulement, réduction des échanges d'eau) et biologique (baisse de la biodiversité et modification de la structure du peuplement de macroinvertébrés) (Wood \& Armitage, 1997; Gayraud et al., 2002). Les communautés de macroinvertébrés benthiques jouent un rôle primordial au sein des écosystèmes d'eau courante où elles occupent une position fondamentale dans les réseaux trophiques (Resh, 2008). Grâce notamment à une importante diversité de profils écologiques, les macroinvertébrés constituent un outil majeur pour l'évaluation de la qualité biologique des cours d'eau et l'identification des différents facteurs de perturbation des milieux intégrables à différentes échelles spatio-temporelles (Statzner et Bêche, 2010). La structure des communautés de macroinvertébrés benthiques est souvent utilisée comme indicateur des effets de l'activité humaine sur les écosystèmes aquatiques, et peut fournir une foule d'informations sur la qualité de l'eau et de l'habitat (Woodcock et Huryn, 2007). Ils sont directement influencés par les conditions physiques de leur milieu de vie comme le colmatage (Gayraud et al., 2002 ; Descloux, 2011, Morel et al., 2015 ). La présente étude vise à évaluer l'impact des eaux usées de la sablière de Toutsang sur la qualité physico-chimique des eaus et sur la biodiversité et la dynamique des macroinvertébrés benthiques du cours d'eau Doulahang. 


\section{Méthodologie}

\section{Zone d'étude et station d'échantillonnage}

Toutsang se situe à $1400 \mathrm{~m}$ d'altitude, à environ $2 \mathrm{~km}$ du centre ville de Dschang. La sablière de Toutsang est située au Sud-Est du centre urbain, à environ ....km du Lycée Bilingue et de l'Université de Dschang. Elle est ouverte sur tout le flanc d'un interfluve en demi orange qui culmine à environ $1457 \mathrm{~m}$ d'altitude. Cette sablière a une hauteur de $57 \mathrm{~m}$ et une superficie d'environ 4,5 ha. Elle est ouverte sur les produits d'altération d'un ortho gneiss et présente trois faciès : micro grenu, grenu et porphyroïde leucocrate. Sur le plan climatiques, la ville de Dschang est sous l'influence d'un climat de type camerounien, fortement modulé par des hautes altitudes et caractérisé par deux saisons dont une longue saison de pluie allant de mars à octobre ( 8 mois) et une courte saison sèche s'étalant sur 4 mois (de novembre à février). La période la plus arrosée est comprise entre le mois de juin $(243,4 \mathrm{~mm})$ et le mois de septembre $(235,2 \mathrm{~mm})$. Le maximum de précipitation est enregistré au mois d'août $(313,9 \mathrm{~mm})$ et le minimum en décembre $(9,5 \mathrm{~mm})$. La température moyenne est de $21^{\circ} \mathrm{C}$.

Compte tenu de l'objectif général de l'étude, le choix des trois stations de prélèvements (TS1, TS2 et TS3) a été effectué en fonction de 1'activité de la sablière (Figure 1).

Station TS1 $\left(05^{\circ} 25^{\prime} 39.9^{\prime}\right.$ 'N et $\left.010^{\circ} 03^{\prime} 45.7^{\prime \prime} \mathrm{E}\right)$ : Le choix de cette station est primordial, du fait qu'elle est considérée comme station de référence (témoin). Elle est située sur le cours d'eau Douhalang, à environ $100 \mathrm{~m}$ en amont du point de confluence du ruisseau recevant les eaux usées de la sablière. Le substrat ici est constitué majoritairement de vase et de limons. Ombragé, le lit de cette station aux eaux très claires, présente une petite quantité de matières organiques en décomposition (débris de feuilles, branches...).

Station TS2 $\left(05^{\circ} 25^{\prime} 40.4^{\prime}\right.$ 'N et $010^{\circ} 03^{\prime} 46.9^{\prime}$ 'E) : Le ruisseau sur lequel se situe cette station traverse la sablière de Toutsang et ses eaux sont utilisées pour l'extraction du sable. Cette station est située à environs $500 \mathrm{~m}$ en aval de la sablière. L'activité de cette dernière est très importante en amont et leurs déchets sont directement rejetés dans le ruisseau. Cette station est choisie afin d'évaluer l'impact des apports des rejets de la sablière sur le ruisseau. Le substrat est hétérogène, constitué de graviers, de sable, de limons et principalement de vase. Les eaux du ruisseau sont très rouge et trouble. A proximité de cette station, se trouvent quelques exploitations agricoles constituées de petits champs de maïs et de banane plantain. Le bassin versant est peu peuplé.

Station TS3 $\left(05^{\circ} 25^{\prime} 36.9^{\prime} ' \mathrm{~N}\right.$ et $\left.010^{\circ} 03^{\prime} 45.3^{\prime}, \mathrm{E}\right)$ : Elle est située à environ $100 \mathrm{~m}$ en aval du point de confluence du ruisseau recevant les eaux usées de la sablière avec le cours d'eau principal. Cette station a été choisie 
pour mieux évaluer l'impact des apports des rejets de la sablière sur le cours d'eau principal. La végétation riveraine est constituée de petits champs de maïs et des plantes de sissogo. Le substrat est constitué de galets, de graviers, de sable et de vase. Le débit est très important par rapport aux stations précédentes.

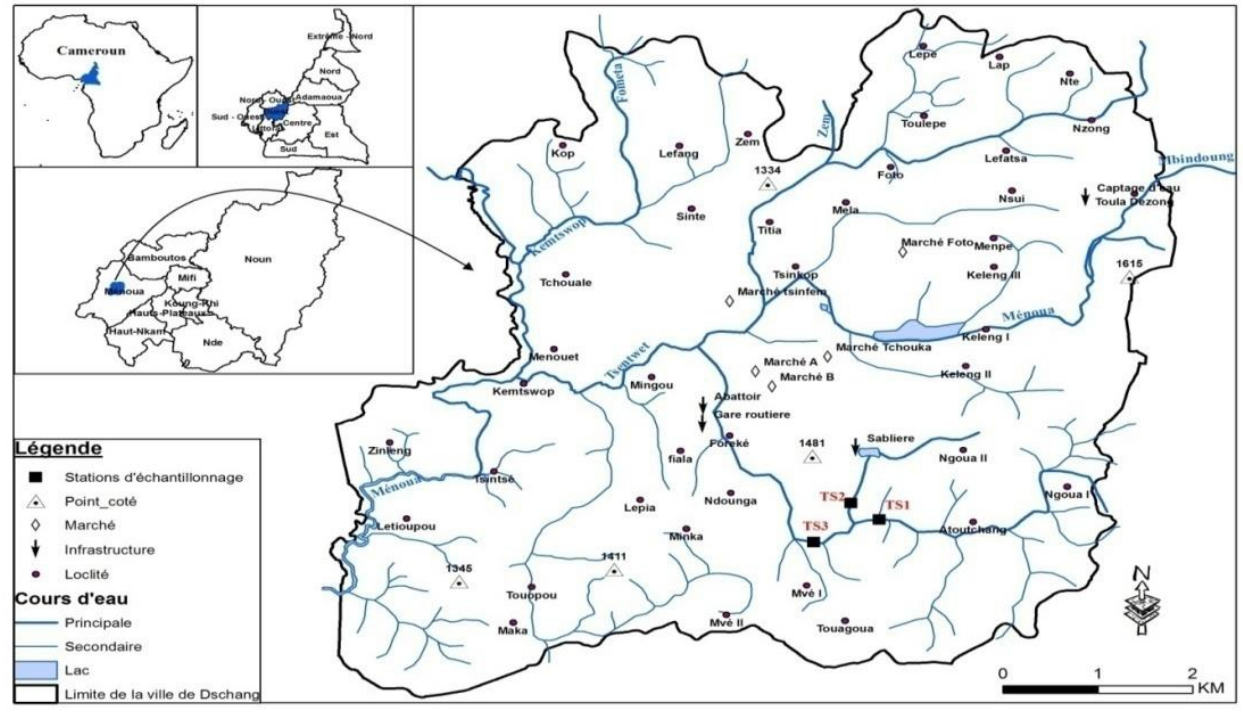

Figure 1: Carte de situation de la zone d'étude et de localisation des stations de prélèvement

\section{Mesure des paramètres hydromorphométriques}

Les mesures des paramètres hydromorphométriques (débit, vitesse moyenne, section mouille, largeur moyenne et profondeur moyenne) ont été effectuées sur le terrain à chaque mois et à chaque station pendant toute la période de l'étude.

La détermination de la vitesse ( $\mathrm{V}$ en $\mathrm{m} / \mathrm{s})$ du courant a été effectuée en surface du cours axial à l'aide d'un flotteur et d'un chronomètre. Le flotteur est lâché en dérive sur une distance (d) connue. Le temps (t) mis par le flotteur à parcourir cette distance a permis de calculer la vitesse par la formule suivante :

$$
\mathrm{V}=\mathrm{d} / \mathrm{t}
$$

L'estimation de la section mouillée s'est faite par la mesure de la largeur moyenne $(\mathrm{L})$ et de la profondeur moyenne $(\mathrm{P})$. La section a été calculée de la façon suivante :

$$
\mathrm{S}=\mathrm{L} \times \mathrm{P}
$$

Le débit $(\mathrm{Q})$ a été donc calculé par la formule :

$\mathrm{Q}=\mathrm{S} \times \mathrm{V}\left(\mathrm{m}^{3} / \mathrm{s}\right)$ où $\mathrm{S}$ est la section mouillée et $\mathrm{V}$ la vitesse moyenne 


\section{Mesures des paramètres physicochimiques}

Les mesures des paramètres physicochimiques se sont déroulées à la fois sur le terrain et au laboratoire suivant les recommandations de APHA (2009) et Rodier et al. (2009). Cinq paramètres physico-chimiques ont été enregistrés sur le terrain: la température, le pH, la salinité, les TDS et la conductivité électrique ont été mesurées par une sonde multiparamètre modèle waterproof IP57.

Pour les paramètres à mesurer au laboratoire (turbidité, nitrates, ammonium, orthophosphates, MES et couleur), les échantillons d'eau ont été prélevés dans des flacons en polyéthylène à double bouchage de $500 \mathrm{ml}$, préalablement rincés avec les eaux du milieu, puis conservés en enceinte réfrigéré. Ces échantillons ont été ensuite transportés au laboratoire de Botanique Appliquée de l'Université de Dschang où les analyses y relatives ont été effectuées suivant les méthodes standards à l'aide d'un spectrophotomètre de marque DR2000 ${ }^{\mathrm{TM}}$.

\section{Echantillonnage, identification et dénombrement des macroinvertébrés benthiques}

Les prélèvements ont été réalisés de mars 2015 à février 2016 (12 mois), suivant une fréquence d'échantillonnage mensuelle. L'échantillonnage a été effectué à l'aide d'un troubleau constitué d'un filet conique de $400 \mu \mathrm{m}$ de vide de maille et $50 \mathrm{~cm}$ de profondeur fixé sur un cadre métallique de $30 \mathrm{~cm}$ de côté, monté sur une manche en acier de $150 \mathrm{~cm}$ de long. Il s'est fait suivant l'approche multihabitat proposée par Stark et al. (2001), adaptée de Babour et al. (1999), qui consiste à effectuer dans chaque station, à chaque campagne, un total de 20 coups de filet dans différents micro-habitats caractérisés par le couple substrat/vitesse. Cette méthode consiste à déposer le cadre du troubleau à même le substrat et balayer le fond de l'eau par des mouvements de va et vient sur une distance de $50 \mathrm{~cm}$. Les organismes ainsi récoltés sont fixés au formol à $10 \%$ sur le terrain. Au laboratoire, les spécimens sont rincés sur un tamis de $400 \mu \mathrm{m}$ d'ouverture de maille puis conservés dans de $1^{\prime}$ alcool à $70^{\circ}$.

La totalité des spécimens a été identifiée jusqu'au plus petit rang taxonomique possible, sous un stéréoscope modèle ZEISS discovery V8, , ceci à l'aide des clés de détermination appropriées. Parmi les clés utilisées, celles de Durand et Lévêque (1981) et Tachet et al. (2010) traitent des invertébrés de façon générale. Les autres clés traitent spécialement des insectes (Dejoux et al., 1981 ; Diomandé et al., 2000 ; Day et al., 2002 ; De Moor et al., 2003 ; Stals \& De Moor, 2007), des crustacés (Day et al., 2001), des mollusques (Brown, 1994 ; De Moor \& Day, 2002). 


\section{Analyse de la structure du peuplement}

La structure du peuplement des macroinvertébrés benthiques dans chaque station a été étudiée à l'aide des métriques tels que : la richesse taxonomique totale, la richesse taxonomique des Diptères, la richesse taxonomique des ETO (Ephéméroptère-Trichoptère-Odonate), la richesse taxonomique des Insectes, l'abondance totale, l'abondance relative des Ephéméroptères-Trichoptère-Odonate $(\% \mathrm{ETO})$, l'abondance relative des Insectes. L'indice de diversité de Shannon et Weaver et l'équitabilité de Pielou ont été également calculés dans chaque station afin d'établir et de comparer la structure du peuplement de macroinvertébrés benthiques d'une station à $1^{\prime}$ 'autre.

Le test ANOVA à un facteur a permis de comparer, les variations des

paramètres physico-chimiques entre les différentes stations d'échantillonnage. Le calcul du coefficient de corrélation de Pearson a permis d'établir les corrélations entre les variables physico-chimiques et biologiques. L'analyse des données a été faite en utilisant le logiciel SPSS version 9.0 .

\section{Résultats}

\section{Variables hydromorphométriques}

Les résultats des paramètres hydromorphométriques mesurés dans les trois stations d'étude sont représentés ci-dessous (tableau 1).

A l'exception de la vitesse qui est de $0,55 \pm 0,15 \mathrm{~m} / \mathrm{s}$, TS2 a les plus faibles valeurs de paramètres hydrologiques. La station TS1 a enrégistré les plus grandes valeurs de la profondeur $(0,65 \pm 0,13 \mathrm{~m})$ et de la largeur du lit $(2,63 \pm 0,41 \mathrm{~m})$, et la plus faible valeur de la vitesse $(0,28 \pm 0,09 \mathrm{~m} / \mathrm{s})$. La station TS3 par contre enregistre les plus grandes valeurs de la vitesse $(0,61 \pm 0,18 \mathrm{~m} / \mathrm{s})$ et du débit $\left(0,73 \pm 0,38 \mathrm{~m}^{3} / \mathrm{s}\right)$.

Tableau 1 : Valeurs moyennes et écart-types des paramètres hydromorphologiques mesurés au niveau des 3 stations d'échantillonnage (TS1, TS2 et TS3) pendant la période d'étude.

\begin{tabular}{ccccc}
\hline & \multicolumn{4}{c}{ Paramètres } \\
\cline { 2 - 5 } Stations & Profondeur $(\mathrm{m})$ & Largeur $(\mathrm{m})$ & Vitesse $(\mathrm{m} / \mathrm{s})$ & Débit $\left(\mathrm{m}^{3} / \mathrm{s}\right)$ \\
\hline TS1 & $0,65 \pm 0,13$ & $2,63 \pm 0,41$ & $0,28 \pm 0,09$ & $0,56 \pm 0,34$ \\
TS2 & $0,19 \pm 0,14$ & $1,19 \pm 0,27$ & $0,55 \pm 0,15$ & $0,14 \pm 0,15$ \\
TS3 & $0,54 \pm 0,24$ & $2,20 \pm 0,29$ & $0,61 \pm 0,18$ & $0,73 \pm 0,38$ \\
\hline
\end{tabular}

\section{Variables physico-chimiques}

L'évolution spatio-temporelle des paramètres physico-chimiques enregistrés pendant toute la période d'étude est représentée à la figure 2 .

les valeurs du $\mathrm{pH}$ s'échelonnent entre 5,92 (station TS3) et 7,60 (station TS1) (Figure $2 \mathrm{~A}$ ). Elles ont été légèrement supérieures à 7 entre les mois de mars et juin dans toutes les stations, indiquant une tendance des eaux 
vers la basicité. Par contre, les valeurs observées pendant les mois de juillet à février ont été inférieures à 7 dans toutes les stations. D'une manière générale, au niveau de chaque station, les moyennes de $\mathrm{pH}$ indiquent une tendance à l'acidité. Le test ANOVA $1(\mathrm{p}>0,05)$ ne montre aucune différence significative entre les valeurs obtenues au niveau des 3 stations.

Durant toute la période d'étude, la température a assez varié (Figure 2 B). La valeur la plus faible $\left(20,3^{\circ} \mathrm{C}\right)$ a été enregistrée à la station TS3 au mois de juin, tandis que la valeur la plus élevée $\left(25,7^{\circ} \mathrm{C}\right)$ a été observée à la station TS2 au mois de février. Globalement, les valeurs de la température ont été significativement $(\mathrm{p}<0,05)$ plus élevées à la station TS2. .

Les valeurs de la conductivité électrique sont comparables dans les stations TS1 et TS3 (figure $2 \mathrm{C}$ ). Elles ont été plus élevées à la station TS2 pendant toute la période de l'étude. La plus faible valeur a été enregistrée aux mois d'octobre et novembre à la station TS1 $(16 \mu \mathrm{S} / \mathrm{cm})$ et la valeur la plus élevée $(\mathrm{p}<0,05)$ a été observée à la station TS2 au mois d'avril (52 $\mu \mathrm{S} / \mathrm{cm})$. Les stations TS1 et TS2 ont respectivement la valeur moyenne la plus faible $(22,31 \pm 31 \mu \mathrm{S} / \mathrm{cm})$ et la valeur moyenne la plus élevée $(33,54 \pm 4,64 \mu \mathrm{S} / \mathrm{cm})$. Au même titre que la tempéruture, aucune différence significative n'a été notée entre les stations TS1 et TS3 ( $\mathrm{p}>0,05)$.

Les valeurs de TDS ont varié d'une station à l'autre et même au niveau d'une même station (Figure 2 D). Les valeurs les plus élevée (68 $\mathrm{mg} / \mathrm{l}$ ) ont été obtenues aux stations TS2 et TS3 au mois de septembre. La valeur la plus faible $(6 \mathrm{mg} / \mathrm{l})$ a été enregistrée à la station TS3 au mois d'octobre. Aucune différence significative $(p>0,05)$ n'est observée entre les valeurs notées au niveau de ces 3 stations.

Le profil de la salinité présente peu de variation entre les stations TS1 et TS3 (Figure 2 E). Par contre, une large variation a été observée entre ces deux stations et la station TS2. Cette station a enregistré les valeurs relativement élevée. Les valeurs de ce paramètre fluctue entre 7 et $57 \mathrm{mg} / \mathrm{l}$ respectivement au niveau de la station TS1 au mois de décembre et TS2 au mois de septembre. Des différences significatives $(\mathrm{p}<0,05)$ sont notées au niveau des variations des valeurs entre les trois stations.

Les teneurs en nitrate ont largement varié dans le temps (Figure 2 F). Dans l'espace, la variation est relativement faible. La Station TS3 a enregistré la plus forte teneur en nitrate $(8,4 \mathrm{mg} / \mathrm{l})$ en octobre tandis que la valeur la plus basse a été observée dans toutes les stations. Les variations de la teneur en nitrate dans les trois stations sont non significatives $((p>0,05)$.

Les valeurs de 1'azote ammoniacal ont varié de $0,01 \mathrm{mg} / 1 \mathrm{au}$ mois de mai en TS1 à 3,54 mg/l au mois de novembre en TS2 (Figure 2 G). La station TS2 a enregistré les valeurs les plus élevées en fonction du temps pendant toute la période de l'étude $(\mathrm{p}<0,05)$. Les différences entre les stations TS1 et TS3 étant non significatives. 
Les profils de variation des MES, de la turbidité et de la couleur sont relativement similaires et présentent des valeurs très faibles à la station TS1 et très élevées à la station TS2 (Figure $2 \mathrm{H}$, I et J). Ces valeurs varient largement d'une station à l'autre et même d'un mois à l'autre. Les stations TS1 et TS2 ont respectivement les valeurs moyennes les plus faibles et les plus élevées de ces trois paramètres. Les valeurs de la turbidité s'échelonnent entre 0NTU (TS1, avril) et 1715NTU (TS2, juin) . Les valeurs de la turbidité obtenues à TS2 sont significativement supérieures $(\mathrm{p}<$ $0,05)$ à celles obtenues dans les autres stations. En ce qui concerne les MES, des très faibles valeurs $(0,0 \mathrm{mg} / \mathrm{l})$ ont été enrégistrée aux mois d'avril et février. Le mois de juin a enrégistrée la plus grande valeur $(3655,0 \mathrm{mg} / \mathrm{l})$. Les valeurs obtenues chevauchent autour d'une moyenne de 11,75 $\pm 11,68$ NTU, 670,0 $\pm 992,19 \mathrm{NTU}, 102,67 \pm 150,90 \mathrm{NTU}$ respectivement à la station TS1, TS2, TS3. Ce paramètre ne présente pas une différence significative ( $p$ $>0,05)$ entre les stations TS1 et TS3. Par contre, une difference significative $(\mathrm{p}<0,05)$ a été observée entre les stations TS1 et TS2 d'une part et entre les stations TS2 et TS3 d'autre part. La couleur a varié entre 11,0 PtCo en février à la station TS3 et 1500,0 PtCo en TS2. Des différences significatives $(\mathrm{p}<0,05)$ sont enrégistrées au niveau des trois stations.

Les teneurs de l'orthophosphaste ont assez varié d'une station à l'autre pendant toute la période de l'étude (Figure $2 \mathrm{~K}$ ). Les plus faibles valeurs $(0,0 \mathrm{mg} / \mathrm{l})$ ont été enrégistrées aux mois de septembre et octobre respectivement aux stations TS2 et TS3. La plus grande valeur a été obtenue à la station TS3 en mars. Aucune différence significative $(p>0,05)$ n'est observée au niveau des valeurs entre ces trois stations. 

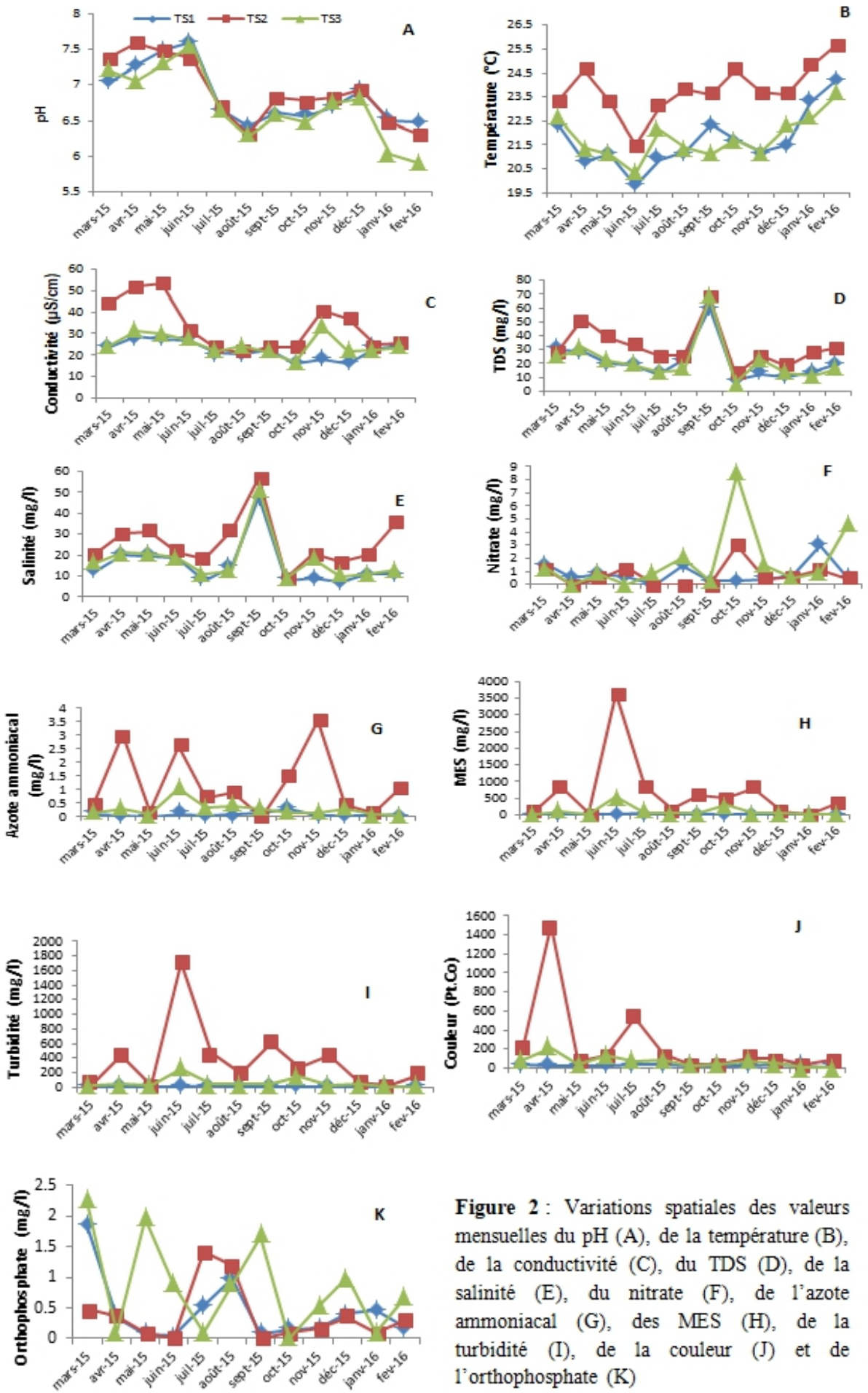

Figure 2: Variations spatiales des valeurs mensuelles du pH (A), de la température (B), de la conductivité (C), du TDS (D), de la salinité (E), du nitrate (F), de l'azote ammoniacal (G), des MES (H), de la turbidité (I), de la couleur (J) et de 1'orthophosphate (K) 


\section{Variables biologiques}

Un total de 63 taxons repartis dans 31 familles, 13 ordres, 6 classes et 4 embranchements ont été récoltés au cours de cette étude (Tableau 2). L'embranchement des Arthropodes est le plus riche en taxons, avec 90,48\% de la richesse totale. Ils sont représentés par les Arachnides constitués d'un seul ordre (Araneides) et les insectes constitués de 56 taxons, 26 familles et 7 ordres. L'ordre des odonates est le plus divesifié avec 23 espèces, suivi des hémiptères ( 9 espèces). Les Mollusques (1,59\%) et les Plathelminthes $(1,59 \%)$ constituent les embranchements les moins représentés au cours de cette étude avec un seul taxon chacun. Les mollusques sont représentés par 1'espèce Biomphalaria pfeifferi appartenant à 1'ordre des Basommatophores et à la famille des Planorbidae. Les Plathelminthes quant à eux sont représentés par le genre Dendrocoelum sp. appartenant à l'ordre des Tricladida et à la famille des Dendrocoelidae. Les Annelides (6,34\%) sont constitués de 4 taxons, 3 familles et 3 ordres.

En ce qui concerne les abondances, un total de 784 individus de macroinvertébrés benthiques ont été récoltés. Les arthropodes représentent l'embranchement le plus abondant avec 732 individus, soit 93,36\% de l'abondance totale. Les mollusques montrent l'abondance la plus faible avec 3 individus soit $0,38 \%$ de l'abondance totale. Quants aux Plathelminthes et aux Annélides, ils représentent respectivement 5,35\% et $0,89 \%$ de l'abondance totale. Les odonates et les diptères constituent les ordres les plus abondants avec respectivement $39,16 \%$ et $25,51 \%$ de l'abondance totale. Ils sont suivies par l'ordre des hémiptères (14,67\%). La variation spatiale des abondances montre que les familles de Coenagrionidae et de Chironomidae sont les plus abondantes au niveau des stations TS3 et TS1 avec respectivement $25,31 \%$ et $14,51 \%$ de l'abondance relative en TS3, $18,28 \%$ et $22,58 \%$ en TS1. La famille des gerridae est la mieux représentée à la station TS2 avec 56,81\% de l'abondance relative.

La distribution spatiale des macroinvertébrés benthiques revèle qu'un nombre élevé de taxons a été enrégistrée au niveau des stations TS1 et TS3 avec respectivement 46 et 47 taxons. Par contre, seulement 18 taxons ont été enrégistrées au niveau de la station TS2 située en aval de la sablière qui rejete les déchets de ses activités directement dans le ruisseau. Le profil d'évolution de l'abondance des macroinvertébrés benthiques est relativement similaire à celui de la richesse spécifique. Avec 372 individus soit 47,45\% de l'abondance globale, la station TS1 présente la plus grande abondance, suivie de la station TS3 avec 324 individus soit 41,33\% de l'abondance totale. La Station TS2 est la moins abondante avec 88 individus soit en moyenne $11,22 \%$ de l'abondance totale. 
Tableau 2 : Liste des taxa de la macrofaune benthique récoltés au niveau des 3 stations d'échantillonnage durant la période d'étude ; $n d=$ taxon non déterminé

\begin{tabular}{|c|c|c|c|c|c|c|c|c|}
\hline $\begin{array}{c}\text { Embranch } \\
\text { ements }\end{array}$ & Classes & Ordres & Familles & Genres ou espèces & $\begin{array}{c}\mathrm{TS} \\
1 \\
\end{array}$ & $\begin{array}{c}\mathrm{TS} \\
2 \\
\end{array}$ & $\begin{array}{c}\text { TS } \\
3\end{array}$ & $\begin{array}{c}\text { Tot } \\
\text { al }\end{array}$ \\
\hline \multirow{43}{*}{$\begin{array}{l}\text { Arthropo } \\
\text { des }\end{array}$} & \multirow{43}{*}{ Insectes } & \multirow{9}{*}{$\begin{array}{l}\text { Hémip } \\
\text { tères }\end{array}$} & \multirow{3}{*}{ Naucoridae } & Naucoris maculatus & 9 & 0 & 11 & 20 \\
\hline & & & & Naucoris sp. & 2 & 0 & 1 & 3 \\
\hline & & & & Neomacrocoris sp. & 0 & 0 & 2 & 2 \\
\hline & & & \multirow{3}{*}{ Népidea } & Nepa sp. & 0 & 2 & 0 & 2 \\
\hline & & & & Laccotrephes sp. & 1 & 0 & 1 & 2 \\
\hline & & & & Ranatra linearis & 2 & 0 & 1 & 3 \\
\hline & & & \multirow{2}{*}{ Veliidae } & Rhagovelia reitteri & 0 & 0 & 2 & 2 \\
\hline & & & & Rhagovelia sp. & 1 & 7 & 1 & 9 \\
\hline & & & Gerridae & Eurymetra sp. & 13 & 50 & 9 & 72 \\
\hline & & \multirow{23}{*}{$\begin{array}{l}\text { Odona } \\
\text { tes }\end{array}$} & \multirow{3}{*}{ Corduliidae } & $\begin{array}{c}\text { Phyllomacromia } \\
\text { picta }\end{array}$ & 4 & 0 & 1 & 5 \\
\hline & & & & Hemicordulia sp. & 0 & 0 & 1 & 1 \\
\hline & & & & Cordulia aenea & 1 & 0 & 0 & 1 \\
\hline & & & \multirow{2}{*}{$\begin{array}{c}\text { Coenagrionida } \\
\mathrm{e}\end{array}$} & Pseudagrion sp. & 48 & 3 & 58 & 109 \\
\hline & & & & Coenagrion proparte & 20 & 0 & 24 & 44 \\
\hline & & & \multirow{2}{*}{$\begin{array}{c}\text { Calopterygida } \\
\mathrm{e}\end{array}$} & Phaon iridipennis & 2 & 1 & 2 & 5 \\
\hline & & & & Calopteryx & 1 & 0 & 0 & 1 \\
\hline & & & \multirow{9}{*}{ Libellulidae } & Rhyothemis sp. & 8 & 0 & 1 & 9 \\
\hline & & & & Zygonyx sp. & 13 & 1 & 23 & 32 \\
\hline & & & & Orthetrum & 12 & 1 & 18 & 31 \\
\hline & & & & Tetrathemis sp. & 7 & 0 & 2 & 9 \\
\hline & & & & Trithemis sp. & 1 & 0 & 0 & 1 \\
\hline & & & & Rhyothemis & 3 & 0 & 0 & 3 \\
\hline & & & & $\begin{array}{c}\text { Brachythemis } \\
\text { leucosticta }\end{array}$ & 1 & 0 & 10 & 11 \\
\hline & & & & $\begin{array}{c}\text { Sympetrum } \\
\text { fonscolombii }\end{array}$ & 11 & 1 & 5 & 17 \\
\hline & & & & Notiothemis sp. & 0 & 0 & 1 & 1 \\
\hline & & & \multirow[t]{2}{*}{ Lestidae } & Sympecma sp. & 5 & 0 & 1 & 6 \\
\hline & & & & Lestes sp. & 2 & 0 & 2 & 4 \\
\hline & & & \multirow[t]{4}{*}{ Gomphidae } & Gomphus sp. & 4 & 0 & 1 & 5 \\
\hline & & & & Paragomphus sp. & 1 & 0 & 0 & 1 \\
\hline & & & & Lestinogomphus sp. & 0 & 0 & 1 & 1 \\
\hline & & & & Phllogomphus sp. & 3 & 0 & 1 & 4 \\
\hline & & & Macromlidae & Macromia splendes & 1 & 0 & 0 & 1 \\
\hline & & \multirow{3}{*}{$\begin{array}{l}\text { Tricho } \\
\text { ptères }\end{array}$} & \multirow{2}{*}{$\begin{array}{l}\text { Hydropsychid } \\
\text { ae }\end{array}$} & Hydropsyche sp & 1 & 0 & 17 & 18 \\
\hline & & & & Cheumatopsyche sp. & 0 & 0 & 2 & 2 \\
\hline & & & $\begin{array}{c}\text { Glossosomati } \\
\text { dae }\end{array}$ & Agapetus agilis & 1 & 0 & 0 & 1 \\
\hline & & \multirow{8}{*}{$\begin{array}{l}\text { Diptèr } \\
\text { es }\end{array}$} & Sciomyzidae & nd. & 1 & 0 & 4 & 5 \\
\hline & & & \multirow[t]{2}{*}{ Chironomidae } & $\begin{array}{l}\text { nd. Chironominae } \\
\text { (SF) }\end{array}$ & 71 & 5 & 43 & 119 \\
\hline & & & & Chironomus sp. & 13 & 2 & 4 & 19 \\
\hline & & & Tipulidae & Tipula sp. & 16 & 4 & 24 & 44 \\
\hline & & & Syrphidae & nd & 1 & 0 & 0 & 1 \\
\hline & & & $\begin{array}{c}\text { Ceratogoponi } \\
\text { dae }\end{array}$ & Bezzia sp. & 2 & 0 & 1 & 3 \\
\hline & & & \multirow[t]{2}{*}{ Simulidae } & nd. & 5 & 0 & 2 & 7 \\
\hline & & & & Paracnephia sp. & 2 & 0 & 0 & 2 \\
\hline
\end{tabular}




\begin{tabular}{|c|c|c|c|c|c|c|c|c|}
\hline & & & Baetidae & Baetis sp. & 4 & 1 & 16 & 21 \\
\hline & & & Leptophlebiid & Leptophlebia sp. & 5 & 0 & 2 & \\
\hline & & & ae & $\begin{array}{c}\text { Adenophlebia } \\
\text { auriculata }\end{array}$ & 7 & 0 & 1 & 8 \\
\hline & & Ephé & & $\begin{array}{c}\text { Adenophlebia } \\
\text { Sylvatica }\end{array}$ & 2 & 0 & 0 & 2 \\
\hline & & mérop & Caenidae & Caenis sp. & 1 & 0 & 1 & 2 \\
\hline & & tères & Siphlonuridae & Siphlonurus sp. & 0 & 0 & 2 & 2 \\
\hline & & & Gyrinidae & $\begin{array}{l}\text { Orectogyrus } \\
\text { specularis }\end{array}$ & 11 & 7 & 4 & 22 \\
\hline & & & & Aulonogyrus sp. & 0 & 1 & 0 & 1 \\
\hline & & & & Hydrochara sp. & 0 & 0 & 1 & 1 \\
\hline & & Coléo & & $\begin{array}{l}\text { Hydrophilus } \\
\text { senegalensis }\end{array}$ & 13 & 0 & 5 & 18 \\
\hline & & ptères & Hydrophilidae & Laccobias sp. & 0 & 0 & 1 & 1 \\
\hline & & & & Hydrophilinae $(S F)$ & 0 & 0 & 2 & 2 \\
\hline & & $\begin{array}{c}\text { Plécop } \\
\text { tères }\end{array}$ & Neoperlidae & Neoperla sp. & 0 & 1 & 0 & 1 \\
\hline & $\begin{array}{c}\text { Arachni } \\
\text { des }\end{array}$ & $\begin{array}{c}\text { Aranei } \\
\text { des }\end{array}$ & nd & nd & 0 & 1 & 0 & 1 \\
\hline & Achète & $\begin{array}{c}\text { Rhync } \\
\text { hobdel } \\
\text { lida }\end{array}$ & $\begin{array}{c}\text { Glossiphoniid } \\
\text { ae }\end{array}$ & Haementeria costata & 0 & 0 & 2 & 2 \\
\hline & $\begin{array}{l}\text { Oligoch } \\
\text { ètes }\end{array}$ & $\begin{array}{c}\text { Haplot } \\
\text { axida }\end{array}$ & Tubificidae & Branchiura sp. & 0 & 0 & 2 & 2 \\
\hline & & & & nd. & 2 & 0 & 0 & 2 \\
\hline Annélides & & $\begin{array}{l}\text { Lumbr } \\
\text { icida }\end{array}$ & Lumbricidae & Eisniella tetraedra & 1 & 0 & 0 & 1 \\
\hline $\begin{array}{l}\text { Mollusqu } \\
\text { es }\end{array}$ & $\begin{array}{l}\text { Gastéro } \\
\text { podes }\end{array}$ & $\begin{array}{l}\text { Baso } \\
\text { mmat } \\
\text { ophor } \\
\text { e }\end{array}$ & Planorbidae & $\begin{array}{c}\text { Biomphalaria } \\
\text { pfeifferi }\end{array}$ & 0 & 0 & 3 & 3 \\
\hline $\begin{array}{c}\text { Plathelmi } \\
\text { nthes }\end{array}$ & $\begin{array}{c}\text { Turbella } \\
\text { ries }\end{array}$ & $\begin{array}{c}\text { Tricla } \\
\text { dida }\end{array}$ & $\begin{array}{c}\text { Dendrocoelida } \\
\mathrm{e}\end{array}$ & Dendrocoelum sp. & 37 & 0 & 5 & 42 \\
\hline Total & & & & & 46 & 18 & 47 & 63 \\
\hline
\end{tabular}

Les plus faibles valeurs de toutes les métriques calculées ont été enregistrées au niveau de la station TS2 (tableau 3). Cette station reçoit les rejets d'eau usée de la sablière. Les valeurs de l'indice de Shannon Weaver calculées ont oscillé entre 2,48 (TS2) et 4,41 (TS1). Les valeurs de l'équitabilité de Piélou calculées au niveau de chacune des 3 stations étudiées ont varié de 0,62 (station TS2) à 0,80 (station TS1). D'autre métriques telles que la richesse taxonomique des Diptères, la richesse des ETO (Ephéméroptère-Trichoptère-Odonate), la richesse taxonomique des Insectes et l'abondance relative des Ephéméroptères-Trichoptère-Odonate (\%ETO) ont révélé une dégradation de la qualité des eaux et une baisse de la diversité à la station TS2. 
Tableau 3 : valeurs des métriques permettant de décrire la structure des communautés de macroinvertébrés benthiques dans ce tableau, il faut enlever les 00 après la virgule

\begin{tabular}{cccc}
\hline & \multicolumn{3}{c}{ Stations } \\
\cline { 2 - 4 } Métriques & TS1 & TS2 & TS3 \\
\hline RTT & 46 & 18 & 47 \\
RTD & 8 & 3 & 6 \\
ETO & 27 & 6 & 24 \\
RTI & 43 & 14 & 43 \\
\%ETO & 45,43 & 9,09 & 59,57 \\
H“ (bits/ind.) & 4,41 & 2,48 & 4,34 \\
E & 0,80 & 0,62 & 0,78 \\
\hline
\end{tabular}

RTT $=$ Richesse taxonomique totale, $\mathrm{RTD}=$ Richesse taxonomique des Diptères, $\mathrm{ETO}=$ Richesse des Ephéméroptère-Trichoptère-Odonate, RTI = Richesse taxonomique des Insectes, \%ETO = Abondance relative des Ephéméroptères-Trichoptère-Odonate, $\mathrm{H}^{\prime}=$ indice de diversité de Shannon et Weaver, E = Equitabilité de Pielou

La corrélation de Pearson a permit de mettre en évidence une certaine relation entre la qualité des eaux et les métriques descriptrices de la structure des communautés de macroinvertébrés benthiques (Tableau 4). Une corrélation négative et significative a été observée entre les métriques et la température, la conductivité, le TDS, la salinité, l'azote ammoniacal, la turbidité, les MES et la couleur. Elle a été très significative entres les métriques et certains paramètres physico-chimiques tels la température, la conductivité, l'azote ammoniacal, la turbidité et les MES.

Tableau 4 : Valeurs de coefficients de corrélation de Pearson entre les paramètres physicochimiques et les différentes métriques des macroinvertébrés benthiques

\begin{tabular}{|c|c|c|c|c|c|c|c|}
\hline & RTT & RTD & ETO & RTI & \%ЕTO & $\mathbf{H}^{\prime}$ & $\mathbf{E}$ \\
\hline pH & $-0,137$ & $-0,072$ & $-0,116$ & $-0,134$ & $-0,163$ & $-0,130$ & $-0,120$ \\
\hline \multirow[t]{2}{*}{ Température } & - & - & - & - & - & - & - \\
\hline & $0,694^{* *}$ & $0,647^{* *}$ & $0,692^{* *}$ & $0,695^{* *}$ & $0,662^{* *}$ & $0,695^{* *}$ & $0,694^{* *}$ \\
\hline \multirow[t]{2}{*}{ Conductivité } & - & - & - & - & - & _ & - \\
\hline & $0,531^{* *}$ & $0,538^{* *}$ & $0,546^{* *}$ & $0,535^{* *}$ & $0,483^{* *}$ &, $0538^{* *}$ & $0,544^{* *}$ \\
\hline TDS & $-0,330^{*}$ & $-0,308$ & $-0,329$ & $-0,330^{*}$ & $-0,315$ & $-0,331^{*}$ & $-0,330^{*}$ \\
\hline Salinité & $-0,369^{*}$ & $-0,370^{*}$ & $-0,378^{*}$ & $-0,372^{*}$ & $-0,338^{*}$ & $-0,374^{*}$ & $-0,377^{*}$ \\
\hline Nitrates & 0,186 & 0,069 & 0,146 & 0,180 & 0,239 & 0,172 & 0,154 \\
\hline Azote & - & - & - & - & - & - & - \\
\hline ammoniacal & $0,640^{* *}$ & $0,625^{* *}$ & $0,649^{* *}$ & $0,643^{* *}$ & $0,595^{* *}$ & $0,646^{* *}$ & $0,649^{* *}$ \\
\hline \multirow[t]{2}{*}{ Turbidité } & - & - & - & - & - & - & - \\
\hline & $0,518^{* *}$ & $0,504^{* *}$ & $0,525^{* *}$ & $0,520^{* *}$ & $0,483^{* *}$ & $0,522^{* *}$ & $0,524^{* *}$ \\
\hline \multirow[t]{2}{*}{ MES } & - & - & - & - & - & - & - \\
\hline & $0,459^{* *}$ & $0,447^{* *}$ & $0,465^{* *}$ & $0,461^{* *}$ & $0,428^{* *}$ & $0,463^{* *}$ & $0,465^{* *}$ \\
\hline Couleur & $-0,378^{*}$ & $-0,375^{*}$ & $-0,385^{*}$ & $-0,380^{*}$ & $-0,347^{*}$ & $-0,382^{*}$ & $-0,384^{*}$ \\
\hline $\begin{array}{l}\text { Orthophosphate } \\
\text { S }\end{array}$ & 0,209 & 0,078 & 0,164 & 0,201 & 0,267 & 0,193 & 0,173 \\
\hline
\end{tabular}




\section{Discussion \\ Caractéristiques physicochimiques}

Les valeurs moyennes de $\mathrm{pH}$ enregistrées au niveau des 3 stations révèlent une légère tendance des eaux à l'acidité. Ces valeurs peuvent résulter de la nature des terrains traversés par l'eau et du sol de son bassin versant (Rodier et al., 2009). Selon Mumbe (2006), le manteau d'altération des carrières de sable de Menouet et Toutsang est constitué d'une association à base de kaolinite, quartz et de gibbsite.

Les faibles valeurs de température $\left(20,3-25,7^{\circ} \mathrm{C}\right)$ enrégistrées dans les 3 stations pendant la période d'étude pourraient s'expliquer par la localisation de la zone d'étude en milieu périurbaine où la couverture végétale entraine un faible ensoleillement des cours d'eau. A ce sujet, Tchakonté (2016) soulignent que les faibles valeurs de température (24 $27^{\circ} \mathrm{C}$ ) observées dans les stations situées en milieu périurbain résulte du faible ensoleillement du cours d'eau dû à la présence important de la canopée qui constitue une barrière réduisant l'impact des rayons solaires sur la variation de la température de l'eau. La plus grande valeur de la température enrégistrée au niveau de la station TS2 pourraient être due à la faible profondeur de l'eau $(0,18 \mathrm{~m})$.

La faible minéralisation des eaux se traduisent par des très faibles valeurs de la conductivité électrique (16-52 $\mu \mathrm{S} / \mathrm{cm})$, des TDS et de la salinité au niveau des 3stations. Ces valeurs sont similaires à celle obtenues par Madomguia (2009) dans le Nga cours d'eau du milieu non anthropisé. Les valeurs élevées de ces trois paramètres observées au niveau de la station TS2 par rapport à celles de la station TS1, montrent que les rejets de l'eau usée de la sablière auraient un impact direct sur la minéralisation des eaux. Les eaux usées et de ruissellements entrainent une augmentation des valeurs de la conductivité et du taux de solides dissous (Camara et al., 2014). Selon Victor et Onomivbori (1996), les activités anthropiques réalisées à côté des cours d'eau influencent largement la conductivité des eaux des rivières.

Les fluctuations spatio-temporelles de la teneur de l'eau en azote minérale $\left(\mathrm{NH}_{4}{ }^{+}, \mathrm{NO}_{3}{ }^{-}\right)$et en orthophosphates $\left(\mathrm{PO}_{4}{ }^{3-}\right)$ montrent des valeurs faibles excepté la teneur de $\mathrm{NO}_{3}^{-}$enregistrée à la station TS3 au mois d'octobre et la concentration de l'orthophosphate entre mars et septembre. Ceci pourrait être dû à la faible utilisation des engrais et des pesticides dans le bassin versant. Ces résultats s'apparentent à ceux de Tchakonté (2016) et de Foto Menbohan et al. (2013) enregistrés dans des cours périurbains de la ville de Douala et Yaoundé respectivement. Des faibles teneurs en nitrate et orthophosphate avaient également été relevées par Diomandé et al. (2009) dans la rivière Agnéby en Côte d'Ivoire. La concentration élevée de ces deux paramètres résulterait des important lessivages dû aux pluies, car ces mois font parti de la saison pluvieuse à Dschang. 
Les teneurs moyennes les plus élevées des MES, de la couleur et de la turbidité sont enregistrées à la station TS3. Les fortes valeurs de ces paramètres observées au niveau de cette station résulteraient de l'apport de rejets de la sablière. En effet, les eaux chargées de boue issues des activités de la sablière sont permanemment déchargées dans le cours d'eau de la station TS3. Des résultats similaires avaient été observés par Kikouagni (2015) sur la pollution de l'eau en rapport avec l'activité artisanale de la sablière de Fangoum II dans l'arrondissement de Batié.

\section{Caractéristiques biologiques}

Cette étude a permis de recenser 63 taxa de macroinvertébrés benthiques dans l'ensemble des 3 stations étudiées. Ces résultats se rapprochent à ceux de Foto Menbohan et al. (2011) et Tchakonté (2016) qui ont obtenues 57 taxa respectivement dans un cours d'eau anthropisé en milieu tropical et 70 taxa dans les cours d'eau urbains de Douala. La faible richesse taxonomique obtenue serait la conséquence de l'évacuation directe des rejets de l'eau usée de la sablière dans le cours d'eau qui entrainerait le colmatage du lit de ce dernier. De même, les faibles valeurs des métriques enregistrées à la station TS2 indiqueraient l'effet significatif du colmatage sur les assemblages faunistiques benthiques. Ces résultats corroborent ceux de Descloux (2011) qui a montré que la densité, la richesse taxonomique et le pourcentage de taxons EPT diminuent de façon linéaire avec l'augmentation du sédiment fin. Gayraud (2001), a également signalé l'influence de la composition granulométrique sur les densités, la diversité et les traits biologiques invertébrés benthiques. Les fortes abondances enregistrées au niveau des stations TS1 et TS3 résulteraient du fait que les substrats observés au niveau de ces deux stations contiennent essentiellement de la vase et de la matière organique. En effet, selon Chamley et Deconinck (2011), les concentrations en matière organique sont plus élevées dans les sédiments fins que dans les sédiments plus grossiers comme le sable. Ce qui justifie une abondance brute plus élevée des macroinvertébrés benthiques au niveau des stations TS1 et TS3 qu'au niveau de la station TS2 ; la matière organique étant un des maillons de la chaîne trophique de ces derniers. Les faibles valeurs des métriques observées à la station TS2 pourraient également s'expliquer par les conséquences directes du colmatage sur la disponibilité de l'habitat pour les organismes vivants. Les apports excessifs des sédiments fins conduisent à une homogénéisation de l'habitat de la zone benthique et à une diminution importante de la richesse taxonomique (Waters, 1995). La diminution à la station TS2 de la richesse taxonomique des Diptères et l'absence des Oligochètes qui sont des organismes vermiformes souvent perçus comme adaptés à l'enfouissement dans les sédiments fins serait dû à la faible teneur en matières organiques des sédiments et à la faible diversité 
des ressources trophiques (Descloux, 2011). Une autre étude menée par cet auteur a révélé que les valeurs des métriques telles la richesse taxonomique, l'abondance des insectes, des diptères, des Ephéméroptères-TrichoptèresOdonates et de la diversité diminuent avec l'augmentation du colmatage. Les fortes valeurs de l'indice de Shannon et weaver notée au niveau des stations TS1 et TS3 montrent que ces deux stations sont en bonne santé écologique. Les valeurs de l'indice d'équitabilité de Piélou élevée aux stations TS1 et TS3 montrent que la répartition des taxons des macroinvertébrés benthiques est plus équilibrée au niveau de ces deux stations qu'à la station TS2. Les valeurs élevées de ces deux indices ont déjà été observées dans les zones peu ou non perturbées par les activités anthropiques (Tshijik et al., 2015 ; Karrouch et Chahlaoui, 2009 ; Zirirane et al., 2014 ; Tshijik et al., 2016 ; Onana et al., 2016; Tchakonté, 2016). Les eaux usées de la sablière affecteraient les macroinvertébrés benthiques et entraineraient par conséquent une baise de la diversité et de l'équitabilité au niveau de la station TS2.

\section{Conclusion}

Dans cette étude il était question de montrer l'impact des rejets de la sablière de Toutsang sur la qualité physico-chimique et biologique des eaux du cours d'eau Doulahang. Ce travail a permis de faire un premier inventaire des macroivertébrés de la rivière Doulahang comprenant 63 taxons repartis dans 31 familles, 13 ordres, 6 classes et 4 embranchements. Les plus faibles valeurs de la richesse taxonomique totale (18 taxons), de l'abondance (88 individus), de la richesse et de l'abondance des groupes polluo-sensibles (ETO) ont été enregistrées à la station TS2 située en aval de la sablière qui rejete les déchets de ses activités directement dans le ruisseau. Au niveau de cette station, les fortes valeurs des paramètres indicateurs de la pollution physique de l'eau tels que la température, la turbidité, les MES et la couleur ont été observées. L'utilisation des analyses des paramètres physicochimiques des eaux et la détermination des indices biotiques et des métriques permettant de décrire la structure des communautés des macroinvertébrés benthiques ont permis de mettre en évidence la dégradation de la qualité des eaux de la rivière Doulahang par les rejets d'eaux usées de la sablière. Afin d'éviter le colmatage du lit de cette rivière, l'amélioration de la qualité des eaux est nécessaire et passe par la construction d'une station d'épuration des eaux usées de rejets de la sablière.

\section{References:}

1. APHA, (2009). Standard method for examination of water and wastewater. American Public Health Assocation, 20th edition, Washington, DC, 1150p. 
2. Babour M.T., Gerritsen J., Synder B.D. \& Stribling J.B., (1999). Rapid biossessment protols for use in stream and wadeable rivers : periphyton, benthic macroinvertebrates and fish. $2^{\text {nd }}$ edition, U.S. Environmental Protection Agency, office of water, Washington, D.C., EPA $841-B$ - 99-002. 11 chapiters and appendices, $664 p$.

3. Bilotta G.S. et Brazier R.E. , (2008). Understanding the influence of suspended solids on water quality and aquatic biota. Water Research, $n^{\circ} 42$, pp. 2849-2861.

4. Bo T., Fenoglio S., Malacarne G., Pessino M. et Sgariboldi F., (2007). Effects of clogging on stream macroinvertebrates: An experimental approach. Limnologica-Ecology and Mangement of Inland Waters, $n^{\circ} 37, \mathrm{pp}$. 186-192.

5. Brown D.S., (1994). Freshwater Snails of Africa and their Medical Importance. CRC Press, Taylor \& Francis Ltd. Ed, London, $2^{\text {nd }}$ revised edition, 609p.

6. Camara A.I, Diomande D. \& Gourene G., (2014). Impact des eaux usées et de ruissellement sur la biodiversité des macroinvertébrés de la rivière banco (parc national du banco ; Côte d'Ivoire), $R E V$. CAMES, Vol. 02.

7. Chamley H., Deconinck J.F., (2011). Bases de Sédimentologie (3ème édn). Dunod: Paris.

8. Day J.A., Harrison A.D. \& De Moor I.J., (2002). Guides to the Freshwater Invertebrates of Southern Africa, Volume 9 : Diptera. Water Research Commission Report, No. TT 201/02, Pretoria-South Africa, 210p.

9. Day J.A., Stewart B.A., De Moor I.J. \& Louw A.E., (2001). Guides to the Freshwater Invertebrates of Southern Africa, Volume 4: Crustacea III. Water Research Commission Report, No. TT 141/101, Pretoria-South Africa, 151p.

10. Dejoux C., Elouard J.M., Forge P. \& Maslin J.L., (1981). Catalogue iconographique des insectes aquatiques de Côte d'Ivoire. ORSTOM 42, 178p.

11. De Moor I.J. \& Day J.A., (2002). Guides to the Freshwater Invertebrates of Southern Africa, Volume 6 : Arachnida \& Mollusca. Chapter 3 : Mollusca. Water Research Commission Report, No. TT 182/02, Pretoria-South Africa, pp. 42-125.

12. De Moor I.J., Day J.A. \& De Moor F.C., (2003). Guides to the Freshwater Invertebrates of Southern Africa, Volume 7 : Insecta I. Ephemeroptera, Odonata \& Plecoptera. Water Research Commission Report, No. TT 207/03, Pretoria-South Africa, 301p.

13. Descloux S., (2011). Le colmatage minéral du lit des cours d'eau : méthode d'estimation et effets sur la composition et la structure des 
communautés d'invertébrés benthiques et hyporhéiques. Thèse de doctorat, université Claude Bernard - Lyon I, 215p.

14. Diomandé D., Bony Y.K., Edia O.E., Konnan K.F., Gourène G., (2009). Diversité des Macroinvertébrés benthiques de la rivière Agnéby (Côte d'Ivoire ; Afrique de l'ouest). European journal of Scientific Research, $n^{\circ} 35$, pp. 328-377.

15. Diomandé D., Gourène G., Sankaré Y. \& Zabi S.G., (2000). Synopsis de la classification des larves et des nymphes de Diptères Chironomidea des écosystèmes dulçaquicoles de l'Afrique de l'Ouest. Clés de détermination des sous-familles, des tribus et des genres. Archives scientifiques $d u$ Centre de Recherches Océanologiques d'Abidjan, $n^{\circ} 17$, pp.1-31.

16. Durand J.R. \& Lévêque C. (1981). Flore et faune aquatique de l'Afrique Sahélo-soudanenne. Tome II. Edition de L'ORSTOM, Paris, 517p.

17. Foto Menbohan S., Tchakonté S., Ajeagah G.A., Zébazé T.S.H., Bilong Bilong C.F. \& Njiné T., (2013). Water quality assessment using benthic macroinvertebrates in a periurban sream (Cameroon). The International Journal of Biotechnology, $n^{\circ} 2$, pp. $91-104$.

18. Foto M.S., Zébazé T.S.H., Nyamsi T.N.L., Ajeagah G.A., Njiné T., (2011). Evolution Spatiale de la diversité des Peuplements de Macroinvertébrés Benthiques dans un cours d'eau Anthropisé en Milieu Tropical (Cameroun). European Journal of Scientific Research, $n^{\circ}$ 55, pp. 291-300.

19. Gayraud S., (2001). Les sédiments du lit des cours d'eau : quantification de leur influence sur les peuplements de macroinvertébrés par une approche multi-sites. Thèse de doctorat. Université Claude Bernard-Lyon 1, 215p.

20. Gayraud S., (2002). Colmatage minéral du lit des cours d'eau : revue bibliographique des mécanismes et des conséauences sur les habitats et les peuplements de macroinvertébrés. Bull. Fr. Pêche Piscic. Pp. $339-355$.

21. Greig S.M., Sear D.A. et Carling P.A., (2005). The impact of fine sediment accumulation on the survival of incubating salmon progeny, Implications for sediment management. Science of the Total Environment, $n^{\circ} 344$, pp. 241-258.

22. Karrouch L., Chahlaoui A., (2009). Bio-evaluation de la qualite des eaux de l'Oued Boufekrane (Meknes, Maroc). Biomatec Echo, $n^{\circ} 3$, рр. $6-17$.

23. Kemp P., Sear D., Collins A., Naden P. et Jones I., (2011). The impacts of fine sediment on riverine fish, Hydrological Processes, Published online in Wiley Online Library. 
24. Kikouagni A., (2015). Evaluation de la pollution de l'eau en rapport avec l'activité artisanale de la sablière de Fangoum II dans l'arrondissement de Batié. Mémoire de Master d'ingénieur en mines et pétrole, université de Dschang, 34p.

25. Morel A., Dumont B., Pujol A., Archambaud-Suard G. et Bêche L., (2015). Dynamique de recolonisation des macroinvertébrés benthiques suite au décolmatage par une crue en Durance régulée.

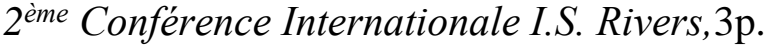

26. Mumbé D.H.T., (2006). Etude minéralogique et propriétés physicochimiques de résidus argileux des sablières de Dschang : importance dans la céramique. Thèse de Master of Science, Université de Dschang, 66p.

27. Onana F.M., Zébazé T.S.H., Koji E., Nyamsi T.N.L. et Tchakonté S., (2016).

28. Influence of municipal and industrial pollution on the diversity and the structure of benthic macro-invertebrates community of an urban river in Douala, Cameroon. Journal of Biodiversity and Environmental Sciences (JBES), $n^{\circ}$ 8, pp.120-133.

29. Rabeni C.F., Doisy K.E. et Zweig L.D., (2005). Stream invertebrate community functional responses to deposited sediment. Aquatic Sciences, $n^{\circ} 67, p p$. 395-402.

30. Resh V.H., (2008). Which group is best? Attributes of different biological assemblages used in freshwater biomonitoring programs. Environmental Monitoring and Assessment $n^{\circ} 138$, pp.131-138.

31. Rodier J., Legube B., Merlet N., (2009). L'analyse de l'eau. Paris, Dunod, 9e édition, $1579 \mathrm{p}$.

32. Stals R. \& De Moor I.J., (2007). Guides to the Freshwater Invertebrates of Southern Africa, Volume 10: Coleoptera. Water Research Commission Report, No. TT 320/07, Pretoria-south Africa, $275 p$.

33. Stark J.D., Boothroyd K.G., Harding J.S., Maxted J.R. \& Scarsbrook M.R., (2001). Protocols for Sampling Macroinvertebrates in Wadeable Streams. New Zealand Macroinvertebrates working group, report no.1, Ministry for the Environment and sustainable Management, fund project no.5103, 57p.

34. Statzner B. et Bêche L.A., (2010). Can biological invertebrate traits resolve effects of multiple stressors on running water ecosystems? Freshwater Biology, $n^{\circ} 55$, pp.80-119.

35. Tachet H., Richoux P., Bournaud M. \& Usseglio-Polatera P., (2010). Invertébrés d'eau douce : systématique, biologie et écologie. CNRS édition, Paris, 588p. 
36. Tchakonté S., (2016). Diversité et structure des peuplements de macroinvertébrés benthiques des cours d'eau urbains et périurbains de Douala (Cameroun). Thèse de Doctorat $/ \mathrm{PhD}$. Université de Yaoundé, 205p.

37. Tshijik J-C. K., Akaibe D., Micha J-C. et Pwema V.K., (2016). Analyse comparative de la diversité taxonomique et de la qualité écologique de l'eau des rivières Lukunga et Mangengenge à Kinshasa. International Journal of Innovation and Applied Studies, $\mathrm{n}^{\circ} 16: 260-271$.

38. Tshijik J-C.K., Ifuta S.N., Mbaya A.N. et Pwema V.K., (2015). Influence du substrat sur la répartition des macroinvertébrés benthiques dans un système lotique : cas des rivières Gombe, Kinkusa et Mangengenge. Int. J. Biol. Chem. Sci., $n^{\circ} 9$, pp. 970-985.

39. Tshijik J-C. K., Akaibe D , Micha J-C. et Pwema V.K., (2016). Analyse comparative de la diversité taxonomique et de la qualité écologique de l'eau des rivières Lukunga et Mangengenge à Kinshasa. International Journal of Innovation and Applied Studies, $n^{\circ} 16, p p .260-271$.

40. Victor R. et Onomivbori O., (1996). The effect of urban perturbation on the benthic macroinvertebrates of a southern Nigeria stream, Perspectives in Tropical limnology, pp. 223-238.

41. Waters T.F., (1995). Sediment in streams : sources, biological effects and control. American Fisheries Society, pp. 1-251.

42. Wood P.J. et Armitage P.D.,(1997). Biological effects of fine sediments in the lotic environment. Environment Management, $n^{\circ} 21$, pp. 203-217.

43. Woodcock T.S. et Huryn A.D., (2007). The response of macroinvertebrate production to a pollution gradient in a headwater stream. Freshwater biology, $n^{\circ}$ 52, pp. 177-196.

44. Zirirane D., Bagalwa J.J, Isumbisho M., Mulengezi M., Mukumba I., Bora M., Mucheso J.M., Lukamba A., Iragi G., Irenge B., Kibangu F. et Kamangala R. (2014). Évaluation comparée de la pollution des rivières Kahuwa et Mpungwe par l'utilisation des macroinvertébrés benthiques. VertigO - la revue électronique en sciences de l'environnement, URL : http://vertigo.revues.org/15365 ; DOI : 10.4000/vertigo. 15365 . 